DPUR/TH/65

March, 2020

\title{
Emergence of Einstein Gravity from Weyl Gravity
}

\author{
Ichiro Oda ${ }^{1}$ \\ Department of Physics, Faculty of Science, University of the Ryukyus, \\ Nishihara, Okinawa 903-0213, Japan
}

\begin{abstract}
It is shown that in a quadratic gravity based on Weyl's conformal geometry, not only the Einstein-Hilbert action emerges but also a Weyl gauge field becomes massive in the Weyl gauge condition, $\tilde{R}=k$, for a Weyl gauge symmetry within the framework of the BRST formalism. We also consider a more general gravitational theory with a scalar field in the Weyl geometry and see that the Einstein-Hilbert action can be induced from spontaneous symmetry breakdown of the Weyl gauge symmetry. Thus, it turns out that Weyl's conformal gravity is quantum mechanically equivalent to Einstein's general relativity plus a massive Weyl gauge field, and the Weyl geometry is free from an infamous "second clock problem" in quantum regime.
\end{abstract}

\footnotetext{
${ }^{1}$ E-mail address: ioda@sci.u-ryukyu.ac.jp
} 


\section{Introduction}

About one hundred years ago, shortly after the appearance of general relativity (GR) by Einstein, Weyl proposed a natural and profound generalization of the Riemann geometry [1]. This generalized geometry essentially includes a vector gauge field called the Weyl gauge field in addition to a conventional metric tensor field, and is nowadays called the Weyl geometry. Weyl's idea is that since the Riemann geometry describes gravitation, a more general affine geometry might describe both gravitation and electromagnetism. Then, just as gravitation can be thought as being due to the path dependence of vectorial directions induced by the metric tensor field, electromagnetism could be considered as being due to the path dependence of vectorial magnitudes induced by the Weyl gauge field.

Unfortunately for Weyl, Einstein immediately objected in a postscript of a Weyl's original paper [1] that in the Weyl gauge theory chemical elements with spectral lines of definite frequency could not exist and the relative frequency of two neighbouring atoms of the same kind would be different in general, depending on their past history, which is obviously in contradiction with observation.

With hindsight, in the Weyl gauge theory, there seem to be two closely related but distinct problems, one of which is that the Weyl gauge field cannot be identified with the electromagnetic potential, thereby nullifying the Weyl theory as a unified theory of gravitation and electromagnetism. The other problem can be paraphrased as the second clock problem: Two clocks synchronized at the same space-time point display different clock rates after traveling along distinct paths and arriving at a common space-time point again in the presence of the Weyl gauge field [2].

With respect to the former problem, quantum mechanics came to the rescue of Weyl's gauge theory. What London noticed [3] is that Weyl's idea should be used in a different manner: A scale factor multiplied under parallel transport of a vector might be changed by a phase factor involving $\hbar$, and it is attached to the quantum-mechanical wave function $\psi$ rather than the metric tensor field. Note that a pure phase shift in the wave function is not physically observable so Einstein's objection can be avoided by quantum mechanics. In this way, Weyl's attempt at combining gravitation with electromagnetism actually ended in failure, but the Weyl theory paved the way 
for the appearance of modern gauge theories [4].

Nevertheless, the original Weyl gauge theory is still physically viable unless one insists on identifying the Weyl gauge field with the electromagnetic gauge field since the electromagnetic field is described in terms of $U(1)$ gauge group whereas the Weyl gauge field is generated by a non-compact Abelian group. In fact, the Weyl theory has been studied from various perspectives for a long time [5]-[23] ${ }^{2}$ and it could be regarded as one of the simplest gravitational theories with a non-compact gauge field.

What then becomes of the latter problem, i.e., the second clock problem? This problem is closely related to the treatment of distance (or proper time) between the space-time point $x^{\mu}$ and its infinitesimal neigborhood $x^{\mu}+d x^{\mu}$. In the Riemann geometry, one can define a line element $d s^{2}=g_{\mu \nu} d x^{\mu} d x^{\nu}$, which is invariant under diffeomorphisms. If we use this line element even in the Weyl geometry, we encounter the second clock problem. However, this line element is not invariant under the Weyl gauge transformation and cannot have any definite physical meaning so it should be modified in such a way that a new line element is invariant under the Weyl gauge transformation as well as diffeomorphisms. In fact, such a line element has been already proposed in Refs. [7, 8, 9]. The idea is to introduce a scalar field $\phi(x)$ of weight -1 into the line element to compensate for the metric tensor of weight 2 and then construct a gauge invariant line element, $d \tilde{s}^{2}=\phi^{2} g_{\mu \nu} d x^{\mu} d x^{\nu}$. With this choice of the line element, we can automatically circumvent the infamous second clock problem that the rate of "clocks" depends on their "history", as will be also discussed in the last section.

Notwithstanding this proposal for the gauge invariant line element, there still remains one question: What is the relation between $d s$ and $d \tilde{s}$ ? We would like to respond to this question that quantum field theory would come the rescue of this issue in a similar way that quantum mechanics came to the rescue of Weyl's gauge theory. Recall that in quantum field theory, one must impose a gauge fixing condition from the beginning to quantize a theory. It is then natural to require that once the Weyl gauge invariance is fixed by a gauge fixing condition, and consequently we have only diffeomorphisms as residual symmetries, the gauge invariant line element $d \tilde{s}$ should be reduced to the line element $d s$ in the Riemann geometry since the gauge fixed theory

\footnotetext{
${ }^{2}$ See [24] for a historical review.
} 
is described by means of the Riemann geometry. Note that this requirement, in turn, gives us information as to the gauge fixing condition for the Weyl gauge symmetry.

In this article, we wish to shed light on the relationship between Einstein's general relativity and the Weyl gauge theory, in particular, a problem of how general relativity with a positive cosmological constant can be generated from the Weyl gauge theory via the gauge fixing procedure of the Weyl gauge invariance in the BRST formalism. This problem is of course not new since starting with an action of quadratic gravity, Weyl himself considered the relation to general relativity by taking what we call the Weyl gauge condition, $\tilde{R}=k$. We would like to extend the Weyl's study to the quantum regime on the basis of the BRST formalism. Moreover, we will deal with a more general Weyl gauge theory involving a scalar field and work with a more general gauge condition, $a \tilde{R}+b \phi^{2}=k$. In this generalized theory, we find that the Higgs potential is naturally generated, thereby triggering spontaneous symmetry breakdown of the Weyl gauge invariance. In the process of obtaining these results, we find that the so-called "the second clock problem" is naturally solved within the framework of quantum field theories.

We close this section with an overview of this article. In Section 2, we briefly review the Weyl geometry and the Weyl gauge theory, details of which can be found in other literatures $[1,4]$. In Section 3, we present a more general action of Weyl's quadratic gravity including a scalar field, and derive field equations from it. In Section 4, we present a gauge invariant line element and take a Weyl gauge condition for the Weyl gauge transformation. It is shown that by a suitable choice of a gauge parameter the Einstein-Hilbert action can be derived from the action of Weyl's quadratic gravity. Moreover, it is shown that in the Einstein or unitary gauge the number of physical degrees of freedom is unchanged before and after spontaneous symmetry breakdown (SSB). In Section 5, we work with a general action and take a more general gauge condition where we see that the SSB of the Weyl gauge symmetry induces the Einstein-Hilbert action and a massive Weyl gauge field at the same time. Section 6 is devoted to the conclusion. 


\section{Review of Weyl conformal geometry}

We briefly review the basic concepts and definitions of the Weyl conformal geometry. ${ }^{3}$ In the Weyl geometry, the Weyl gauge transformation, which is the sum of a local scale transformation for a generic field $\Phi(x)$ and a gauge transformation for the Weyl gauge field $S_{\mu}(x)$, is defined as

$$
\Phi(x) \rightarrow \Phi^{\prime}(x)=e^{w \Lambda(x)} \Phi(x), \quad S_{\mu}(x) \rightarrow S_{\mu}^{\prime}(x)=S_{\mu}(x)-\frac{1}{f} \partial_{\mu} \Lambda(x),
$$

where $w$ is called the "Weyl weight", or simply "weight" henceforth, $f$ is the coupling constant for the non-compact Abelian gauge group, and $\Lambda(x)$ is a local parameter for the Weyl transformation. The Weyl gauge transformation for various fields is explicitly given by

$$
\begin{aligned}
g_{\mu \nu}(x) & \rightarrow g_{\mu \nu}^{\prime}(x)=e^{2 \Lambda(x)} g_{\mu \nu}(x), \quad \phi(x) \rightarrow \phi^{\prime}(x)=e^{-\Lambda(x)} \phi(x), \\
\psi(x) & \rightarrow \psi^{\prime}(x)=e^{-\frac{3}{2} \Lambda(x)} \psi(x), \quad A_{\mu}(x) \rightarrow A_{\mu}^{\prime}(x)=A_{\mu}(x)
\end{aligned}
$$

where $g_{\mu \nu}(x), \phi(x), \psi(x)$ and $A_{\mu}(x)$ are the metric tensor, scalar, spinor, and electromagnetic gauge fields, respectively. The covariant derivative $D_{\mu}$ for the Weyl gauge transformation for a generic field $\Phi(x)$ of weight $w$ is defined as

$$
D_{\mu} \Phi \equiv \partial_{\mu} \Phi+w f S_{\mu} \Phi
$$

which transforms covariantly under the Weyl transformation:

$$
D_{\mu} \Phi \rightarrow\left(D_{\mu} \Phi\right)^{\prime}=e^{w \Lambda(x)} D_{\mu} \Phi .
$$

The Weyl geometry is defined as a geometry with a real symmetric metric tensor $g_{\mu \nu}\left(=g_{\nu \mu}\right)$ and a symmetric connection $\tilde{\Gamma}_{\mu \nu}^{\lambda}\left(=\tilde{\Gamma}_{\nu \mu}^{\lambda}\right)$ which is defined as $^{4}$

$$
\begin{aligned}
\tilde{\Gamma}_{\mu \nu}^{\lambda} & =\frac{1}{2} g^{\lambda \rho}\left(D_{\mu} g_{\nu \rho}+D_{\nu} g_{\mu \rho}-D_{\rho} g_{\mu \nu}\right) \\
& =\Gamma_{\mu \nu}^{\lambda}+f\left(S_{\mu} \delta_{\nu}^{\lambda}+S_{\nu} \delta_{\mu}^{\lambda}-S^{\lambda} g_{\mu \nu}\right)
\end{aligned}
$$

\footnotetext{
${ }^{3}$ See also Refs. [25, 12, 18] for a concise introduction of the Weyl geometry.

${ }^{4}$ We often use the tilde characters to express quantities belonging to the Weyl geometry.
} 
where

$$
\Gamma_{\mu \nu}^{\lambda} \equiv \frac{1}{2} g^{\lambda \rho}\left(\partial_{\mu} g_{\nu \rho}+\partial_{\nu} g_{\mu \rho}-\partial_{\rho} g_{\mu \nu}\right),
$$

is the Christoffel symbol in the Riemann geometry. The most important difference between the Riemann geometry and the Weyl one lies in the fact that in the Riemann geometry the metric condition is satisfied

$$
\nabla_{\lambda} g_{\mu \nu} \equiv \partial_{\lambda} g_{\mu \nu}-\Gamma_{\lambda \mu}^{\rho} g_{\rho \nu}-\Gamma_{\lambda \nu}^{\rho} g_{\mu \rho}=0,
$$

while in the Weyl geometry we have

$$
\tilde{\nabla}_{\lambda} g_{\mu \nu} \equiv \partial_{\lambda} g_{\mu \nu}-\tilde{\Gamma}_{\lambda \mu}^{\rho} g_{\rho \nu}-\tilde{\Gamma}_{\lambda \nu}^{\rho} g_{\mu \rho}=-2 f S_{\lambda} g_{\mu \nu}
$$

where $\nabla_{\mu}$ and $\tilde{\nabla}_{\mu}$ are covariant derivatives for diffeomorphisms in the Riemann and Weyl geometries, respectively. Since the metric condition (7) implies that both length and angle are preserved under parallel transport, Eq. (8) shows that only angle, but not length, is preserved by the Weyl connection.

The general covariant derivative for both diffeomorphisms and the Weyl gauge transformation, for instance, for a covariant vector of weight $w$, is defined as

$$
\begin{aligned}
\mathcal{D}_{\mu} V_{\nu} & \equiv D_{\mu} V_{\nu}-\tilde{\Gamma}_{\mu \nu}^{\rho} V_{\rho} \\
& =\tilde{\nabla}_{\mu} V_{\nu}+w f S_{\mu} V_{\nu} \\
& =\nabla_{\mu} V_{\nu}+w f S_{\mu} V_{\nu}-f\left(S_{\mu} \delta_{\nu}^{\rho}+S_{\nu} \delta_{\mu}^{\rho}-S^{\rho} g_{\mu \nu}\right) V_{\rho} \\
& =\partial_{\mu} V_{\nu}+w f S_{\mu} V_{\nu}-\Gamma_{\mu \nu}^{\rho} V_{\rho}-f\left(S_{\mu} \delta_{\nu}^{\rho}+S_{\nu} \delta_{\mu}^{\rho}-S^{\rho} g_{\mu \nu}\right) V_{\rho} .
\end{aligned}
$$

One can verify that using the general covariant derivative, the following metric condition is satisfied:

$$
\mathcal{D}_{\lambda} g_{\mu \nu}=0 .
$$

Moreover, under the Weyl gauge transformation the general covariant derivative for a generic field $\Phi$ of weight $w$ transforms in a covariant manner as desired:

$$
\mathcal{D}_{\mu} \Phi \rightarrow\left(\mathcal{D}_{\mu} \Phi\right)^{\prime}=e^{w \Lambda(x)} \mathcal{D}_{\mu} \Phi,
$$


because the Weyl connection is invariant under the Weyl gauge transformation, i.e., $\tilde{\Gamma}_{\mu \nu}^{\prime \rho}=\tilde{\Gamma}_{\mu \nu}^{\rho}$.

As in the Riemann geometry, in the Weyl geometry one can also construct a Weyl invariant curvature tensor $\tilde{R}_{\mu \nu \rho}{ }^{\sigma}$ via a commutator of the covariant derivative $\tilde{\nabla}_{\mu}^{5}$

$$
\left[\tilde{\nabla}_{\mu}, \tilde{\nabla}_{\nu}\right] V_{\rho}=\tilde{R}_{\mu \nu \rho}{ }^{\sigma} V_{\sigma}
$$

Calculating this commutator, one finds that

$$
\begin{aligned}
\tilde{R}_{\mu \nu \rho}^{\sigma} & =\partial_{\nu} \tilde{\Gamma}_{\mu \rho}^{\sigma}-\partial_{\mu} \tilde{\Gamma}_{\nu \rho}^{\sigma}+\tilde{\Gamma}_{\mu \rho}^{\alpha} \tilde{\Gamma}_{\alpha \nu}^{\sigma}-\tilde{\Gamma}_{\nu \rho}^{\alpha} \tilde{\Gamma}_{\alpha \mu}^{\sigma} \\
& =R_{\mu \nu \rho}{ }^{\sigma}+2 f\left(\delta_{[\mu}^{\sigma} \nabla_{\nu]} S_{\rho}-\delta_{\rho}^{\sigma} \nabla_{[\mu} S_{\nu]}-g_{\rho[\mu} \nabla_{\nu]} S^{\sigma}\right) \\
& +2 f^{2}\left(S_{[\mu} \delta_{\nu]}^{\sigma} S_{\rho}-S_{[\mu} g_{\nu] \rho} S^{\sigma}+\delta_{[\mu}^{\sigma} g_{\nu] \rho} S_{\alpha} S^{\alpha}\right),
\end{aligned}
$$

where $R_{\mu \nu \rho}{ }^{\sigma}$ is the curvature tensor in the Riemann geometry, and we have defined the antisymmetrization by the square bracket, e.g., $A_{[\mu} B_{\nu]} \equiv$ $\frac{1}{2}\left(A_{\mu} B_{\nu}-A_{\nu} B_{\mu}\right)$. Then, it is straightforward to prove the following identities:

$$
\tilde{R}_{\mu \nu \rho}{ }^{\sigma}=-\tilde{R}_{\nu \mu \rho}{ }^{\sigma}, \quad \tilde{R}_{[\mu \nu \rho]}^{\sigma}=0, \quad \tilde{\nabla}_{[\lambda} \tilde{R}_{\mu \nu] \rho}^{\sigma}=0 .
$$

The curvature tensor $\tilde{R}_{\mu \nu \rho}{ }^{\sigma}$ has 26 independent components, twenty of which are possessed by $R_{\mu \nu \rho}{ }^{\sigma}$ and six by the Weyl invariant field strength $H_{\mu \nu} \equiv$ $\partial_{\mu} S_{\nu}-\partial_{\nu} S_{\mu}$

From $\tilde{R}_{\mu \nu \rho}{ }^{\sigma}$ one can define a Weyl invariant Ricci tensor:

$$
\begin{aligned}
\tilde{R}_{\mu \nu} & \equiv \tilde{R}_{\mu \rho \nu}{ }^{\rho} \\
& =R_{\mu \nu}+f\left(-2 \nabla_{\mu} S_{\nu}-H_{\mu \nu}-g_{\mu \nu} \nabla_{\alpha} S^{\alpha}\right) \\
& +2 f^{2}\left(S_{\mu} S_{\nu}-g_{\mu \nu} S_{\alpha} S^{\alpha}\right)
\end{aligned}
$$

Let us note that

$$
\tilde{R}_{[\mu \nu]} \equiv \frac{1}{2}\left(\tilde{R}_{\mu \nu}-\tilde{R}_{\nu \mu}\right)=-2 f H_{\mu \nu}
$$

\footnotetext{
${ }^{5}$ In this article, we make use of the conventions and notation for the Riemann tensors and the metric signature in the Wald textbook [26], and in particular our sign convention is $\eta_{\mu \nu}=\operatorname{diag}(-1,1,1,1)$.
} 
Similarly, one can define a not Weyl invariant but Weyl covariant scalar curvature:

$$
\tilde{R} \equiv g^{\mu \nu} \tilde{R}_{\mu \nu}=R-6 f \nabla_{\mu} S^{\mu}-6 f^{2} S_{\mu} S^{\mu}
$$

One finds that under the Weyl gauge transformation, $\tilde{R} \rightarrow \tilde{R}^{\prime}=e^{-2 \Lambda(x)} \tilde{R}$ while $\tilde{\Gamma}_{\mu \nu}^{\lambda}, \tilde{R}_{\mu \nu \rho}{ }^{\sigma}$ and $\tilde{R}_{\mu \nu}$ are all invariant.

Even in the Weyl geometry, it is possible to write out a generalization of the Gauss-Bonnet topological invariant which can be described as

$$
\begin{aligned}
I_{G B} & \equiv \int d^{4} x \sqrt{-g} \epsilon^{\mu \nu \rho \sigma} \epsilon_{\alpha \beta \gamma \delta} \tilde{R}_{\mu \nu}^{\alpha \beta} \tilde{R}_{\rho \sigma}^{\gamma \delta} \\
& =-2 \int d^{4} x \sqrt{-g}\left(\tilde{R}_{\mu \nu \rho \sigma} \tilde{R}^{\rho \sigma \mu \nu}-4 \tilde{R}_{\mu \nu} \tilde{R}^{\nu \mu}+\tilde{R}^{2}-12 f^{2} H_{\mu \nu} H^{\mu \nu}\right) \\
& =-2 \int d^{4} x \sqrt{-g}\left(R_{\mu \nu \rho \sigma} R^{\mu \nu \rho \sigma}-4 R_{\mu \nu} R^{\mu \nu}+R^{2}\right) .
\end{aligned}
$$

We close this section by discussing a spinor field as an example of matter fields in the Weyl geometry $[10,11]$. As is well known, to describe a spinor field it is necessary to introduce the vierbein $e_{\mu}^{a}$, which is defined as

$$
g_{\mu \nu}=\eta_{a b} e_{\mu}^{a} e_{\nu}^{b}
$$

where $a, b, \cdots$ are local Lorentz indices taking $0,1,2,3$ and $\eta_{a b}=\operatorname{diag}(-1,1,1,1)$.

Now the metric condition (10) takes the form

$$
\mathcal{D}_{\mu} e_{\nu}^{a} \equiv D_{\mu} e_{\nu}^{a}+\tilde{\omega}_{b \mu}^{a} e_{\nu}^{b}-\tilde{\Gamma}_{\mu \nu}^{\rho} e_{\rho}^{a}=0,
$$

where the general covariant derivative is extended to include the local Lorentz transformation whose gauge connection is the spin connection $\tilde{\omega}^{a}{ }_{b \mu}$ of weight 0 in the Weyl geometry, and $D_{\mu} e_{\nu}^{a}=\partial_{\mu} e_{\nu}^{a}+f S_{\mu} e_{\nu}^{a}$ since the vierbein $e_{\mu}^{a}$ has weight 1 . Solving the metric condition (20) leads to the expression of the spin connection in the Weyl geometry

$$
\tilde{\omega}_{a b \mu}=\omega_{a b \mu}+f e_{\mu}^{c}\left(\eta_{a c} S_{b}-\eta_{b c} S_{a}\right),
$$

where $\omega_{a b \mu}$ is the spin connection in the Riemann geometry and we have defined $S_{a} \equiv e_{a}^{\mu} S_{\mu}$. Then, the general covariant derivative for a spinor field $\Psi$ of weight $-\frac{3}{2}$ reads

$$
\mathcal{D}_{\mu} \Psi=D_{\mu} \Psi+\frac{i}{2} \tilde{\omega}_{a b \mu} S^{a b} \Psi
$$


where $D_{\mu} \Psi=\partial_{\mu} \Psi-\frac{3}{2} f S_{\mu} \Psi$ and the Lorentz generator $S^{a b}$ for a spinor field is defined as $S^{a b}=\frac{i}{4}\left[\gamma^{a}, \gamma^{b}\right]$. Here we define the gamma matrices to satisfy the Clifford algebra $\left\{\gamma^{a}, \gamma^{b}\right\}=-2 \eta^{a b}$. Since the spin connection $\tilde{\omega}^{a}{ }_{b \mu}$ has weight 0 , the covariant derivative $\mathcal{D}_{\mu} \Psi$ transforms covariantly under the Weyl gauge transformation

$$
\mathcal{D}_{\mu} \Psi \rightarrow\left(\mathcal{D}_{\mu} \Psi\right)^{\prime}=e^{-\frac{3}{2} \Lambda(x)} \mathcal{D}_{\mu} \Psi .
$$

Then, the Lagrangian density for a massless Dirac spinor field is of form

$$
\mathcal{L}=\frac{i}{2} e e_{a}^{\mu}\left(\bar{\Psi} \gamma^{a} \mathcal{D}_{\mu} \Psi-\mathcal{D}_{\mu} \bar{\Psi} \gamma^{a} \Psi\right)
$$

where $e \equiv \sqrt{-g}, \bar{\Psi} \equiv \Psi^{\dagger} \gamma^{0}$, and $\mathcal{D}_{\mu} \bar{\Psi}$ is given by

$$
\mathcal{D}_{\mu} \bar{\Psi}=D_{\mu} \bar{\Psi}-\bar{\Psi} \frac{i}{2} \tilde{\omega}_{a b \mu} S^{a b} .
$$

Inserting Eqs. (22) and (25) to the Lagrangian density (24), we find that

$$
\begin{aligned}
\mathcal{L} & =\frac{i}{2} e\left[e_{a}^{\mu}\left(\bar{\Psi} \gamma^{a} \partial_{\mu} \Psi-\partial_{\mu} \bar{\Psi} \gamma^{a} \Psi+\frac{i}{2} \omega_{b c \mu} \bar{\Psi}\left\{\gamma^{a}, S^{b c}\right\} \Psi\right)\right. \\
& \left.+\frac{i}{2} f\left(\eta_{a b} S_{c}-\eta_{a c} S_{b}\right) \bar{\Psi}\left\{\gamma^{a}, S^{b c}\right\} \Psi\right]
\end{aligned}
$$

The last term identically vanishes owing to the relation

$$
\left\{\gamma^{a}, S^{b c}\right\}=-\varepsilon^{a b c d} \gamma_{5} \gamma_{d}
$$

where we have defined as $\gamma_{5}=i \gamma^{0} \gamma^{1} \gamma^{2} \gamma^{3}$ and $\varepsilon^{0123}=+1$. Thus, as is well known, the Weyl gauge field $S_{\mu}$ does not couple minimally to a spinor field $\Psi$. Technically speaking, it is the absence of imaginary unit $i$ in the covariant derivative $D_{\mu} \Psi=\partial_{\mu} \Psi-\frac{3}{2} f S_{\mu} \Psi$ that induced this decoupling of the Weyl gauge field from the spinor field. Without the imaginary unit, the terms including the Weyl gauge field cancel out each other in Eq. (24). In a similar manner, we can prove that the Weyl gauge field does not couple to a gauge field either such as the electromagnetic potential $A_{\mu}$. On the other hand, the Weyl gauge field can couple to a scalar field such as the Higgs field as well as a graviton. In such a situation, we cannot help identifying the Weyl gauge field with an elementary particle that constitutes dark matter. It seems that the Weyl gauge theory was rejected as a unified theory of gravitation and electromagnetism but it has revived as a geometrical theory which predicts the existence of dark matter. 


\section{Classical theory}

In this section, we wish to present the most general action of Weyl's gauge theory involving a scalar field and derive field equations.

One of interesting features in the Weyl gauge theory is that if only the metric tensor is allowed to use for the construction of a gravitational action, the action invariant under the Weyl transformation must be of form of quadratic gravity, but is neither of the Einstein-Hilbert term nor of higher derivative terms such as $\tilde{R}^{3}$. Using the topological invariant (18), one can write down a general action of quadratic gravity, which is invariant under the Weyl transformation, as follows:

$$
S_{Q G}=\int d^{4} x \sqrt{-g}\left(\frac{\xi_{0}}{2} \tilde{C}_{\mu \nu \rho \sigma} \tilde{C}^{\mu \nu \rho \sigma}+\frac{\xi_{1}}{2} \tilde{R}^{2}\right),
$$

where $\xi_{0}$ and $\xi_{1}$ are dimensionless coupling constants. And a generalization

of the conformal tensor, $\tilde{C}_{\mu \nu \rho \sigma}$, in the Weyl geometry is defined as in $C_{\mu \nu \rho \sigma}$ in the Riemann geometry:

$$
\begin{aligned}
\tilde{C}_{\mu \nu \rho \sigma} & \equiv \tilde{R}_{\mu \nu \rho \sigma}-\frac{1}{2}\left(g_{\mu \rho} \tilde{R}_{\nu \sigma}+g_{\nu \sigma} \tilde{R}_{\mu \rho}-g_{\mu \sigma} \tilde{R}_{\nu \rho}-g_{\nu \rho} \tilde{R}_{\mu \sigma}\right) \\
& +\frac{1}{6}\left(g_{\mu \rho} g_{\nu \sigma}-g_{\mu \sigma} g_{\nu \rho}\right) \tilde{R} \\
& =C_{\mu \nu \rho \sigma}+f\left[-g_{\rho \sigma} H_{\mu \nu}+\frac{1}{2}\left(g_{\mu \rho} H_{\nu \sigma}+g_{\nu \sigma} H_{\mu \rho}\right.\right. \\
& \left.\left.-g_{\mu \sigma} H_{\nu \rho}-g_{\nu \rho} H_{\mu \sigma}\right)\right] .
\end{aligned}
$$

This conformal tensor in the Weyl geometry has the following properties:

$$
\tilde{C}_{\mu \nu \rho \sigma}=-\tilde{C}_{\nu \mu \rho \sigma}, \quad \tilde{C}_{\mu \nu \rho}^{\nu}=0, \quad \tilde{C}_{\mu \nu \rho}{ }^{\rho}=-4 f H_{\mu \nu} .
$$

Now, to this quadratic action let us add a kinetic action for the Weyl gauge field and an action including a scalar field $\phi$ of weight -1

$$
\begin{aligned}
S & =\int d^{4} x \sqrt{-g}\left(\frac{\xi_{0}}{2} \tilde{C}_{\mu \nu \rho \sigma} \tilde{C}^{\mu \nu \rho \sigma}+\frac{\xi_{1}}{2} \tilde{R}^{2}-\frac{1}{4} H_{\mu \nu} H^{\mu \nu}\right. \\
& \left.+\frac{\xi_{2}}{2} \phi^{2} \tilde{R}-\frac{1}{2} g^{\mu \nu} D_{\mu} \phi D_{\nu} \phi-\frac{\lambda}{4} \phi^{4}\right),
\end{aligned}
$$


where $\xi_{2}$ and $\lambda$ are also dimensionless coupling constants and $D_{\mu} \phi \equiv \partial_{\mu} \phi-$ $f S_{\mu} \phi$. Provided that only the metric tensor and a scalar field are allowed to use, the action (31) is the most general and renormalizable action in the Weyl geometry, but the first term violates the unitarity [27], so we shall drop this term by taking $\xi_{0}=0$ from now on. The action which we consider in this article, therefore, takes the form

$$
S=\int d^{4} x \sqrt{-g}\left(\frac{\xi_{1}}{2} \tilde{R}^{2}-\frac{1}{4} H_{\mu \nu} H^{\mu \nu}+\frac{\xi_{2}}{2} \phi^{2} \tilde{R}-\frac{1}{2} g^{\mu \nu} D_{\mu} \phi D_{\nu} \phi-\frac{\lambda}{4} \phi^{4}\right) .
$$

From the action (32), let us derive field equations for the Weyl gauge field $S_{\mu}$, the scalar field $\phi$ and the gravitational field $g_{\mu \nu}$. The field equation for the Weyl gauge field is given by

$$
\nabla^{\nu} H_{\mu \nu}=6 f D_{\mu}\left(\xi_{1} \tilde{R}+\frac{6 \xi_{2}+1}{12} \phi^{2}\right) .
$$

The identity $\nabla^{\mu} \nabla^{\nu} H_{\mu \nu}=0$ gives rise to a relation

$$
\nabla^{\mu} D_{\mu}\left(\xi_{1} \tilde{R}+\frac{6 \xi_{2}+1}{12} \phi^{2}\right)=0 .
$$

It is easy to derive the field equation for the scalar field whose result is written as

$$
\frac{6 \xi_{2}+1}{6} \phi \tilde{R}=\frac{1}{6} \phi R-\square \phi+\lambda \phi^{3}
$$

Note that in Eqs. (33), (34) and (35) a specific value $\xi_{2}=-\frac{1}{6}$ leads to some simplication of field equations as previously mentioned by Dirac [6].

Finally, the field equation for the gravitational field gives rise to a little lengthy expression

$$
\begin{aligned}
& \xi_{1} \tilde{R}\left(\tilde{R}_{(\mu \nu)}-\frac{1}{4} g_{\mu \nu} \tilde{R}\right)+\frac{\xi_{2}}{2} \phi^{2}\left(\tilde{R}_{(\mu \nu)}-\frac{1}{2} g_{\mu \nu} \tilde{R}\right) \\
= & \frac{1}{2}\left(H_{\mu \alpha} H_{\nu}{ }^{\alpha}-\frac{1}{4} g_{\mu \nu} H_{\alpha \beta} H^{\alpha \beta}\right)+\frac{1}{2}\left(D_{\mu} \phi D_{\nu} \phi-\frac{1}{2} g_{\mu \nu} D_{\alpha} \phi D^{\alpha} \phi\right)-\frac{\lambda}{8} g_{\mu \nu} \phi^{4} \\
+ & 4 f K\left[\nabla_{\mu} S_{\nu}-\frac{1}{4} g_{\mu \nu} \nabla_{\alpha} S^{\alpha}+2 f\left(S_{\mu} S_{\nu}-\frac{1}{4} g_{\mu \nu} S_{\alpha} S^{\alpha}\right)\right] \\
+ & 6 f\left[\frac{1}{2} g_{\mu \nu} \nabla_{\alpha}\left(K S^{\alpha}\right)-\nabla_{(\mu}\left(S_{\nu)} K\right)\right]+\left(\nabla_{\mu} \nabla_{\nu}-g_{\mu \nu} \square\right) K
\end{aligned}
$$


where we have defined $K \equiv \xi_{1} \tilde{R}+\frac{\xi_{2}}{2} \phi^{2}$ and the symmetrization by the round bracket, e.g., $A_{(\mu} B_{\nu)} \equiv \frac{1}{2}\left(A_{\mu} B_{\nu}+A_{\nu} B_{\mu}\right)$. Taking the trace of this equation, we have

$$
-\frac{\xi_{2}}{2} \phi^{2} \tilde{R}=-\frac{1}{2} D_{\alpha} \phi D^{\alpha} \phi-\frac{\lambda}{2} \phi^{4}+6 f \nabla_{\alpha}\left(K S^{\alpha}\right)-3 \square K .
$$

Here we make use of Eq. (34), which can be rewritten as

$$
\square K=-\frac{1}{12} \square \phi^{2}+2 f \nabla_{\alpha}\left[\left(\xi_{1} \tilde{R}+\frac{6 \xi_{2}+1}{12} \phi^{2} S^{\alpha}\right)\right] .
$$

Substituting Eq. (38) into (37), we can obtain (35). Thus, the field equation for the scalar field $\phi$ is not independent but derived from the other field equations due to the Weyl gauge symmetry.

\section{Weyl gauge}

In order to clarify quantum aspects of a general gauge theory, we must impose a gauge fixing condition from the beginning. In this sense, the gauge fixing conditions play an essential role in quantum field theory and their importance should not be underestimated. For instance, in order to show that an Utiyama's incorrect claim, that is, Weyl's gauge field has a negative energy [7, 9], Hayashi and Kugo quantized the Utiyama's action by imposing the "Einstein gauge condition" $\phi(x)=1$ for the Weyl gauge transformation in a manifestly covariant way, and then proved that the gauge field has a positive energy density, thus having a normal behaviour [11].

Thus far, we are familiar with four kinds of gauge fixing conditions in Weyl gravity, thereby making us easy to understand the connection between the Weyl gravity and Einstein's theory of gravitation. The first gauge fixing condition is called the "Weyl gauge" putting Weyl's scalar curvature to a constant, i.e., $\tilde{R}=k[1]$, which will be discussed in detail in this section. The second gauge fixing condition is called the "Einstein gauge", which sets a scalar field called the measure field by Utiyama to a constant. This gauge fixing will be later discussed in this section as well since the Einstein gauge somewhat resembles the "unitary gauge" in the Higgs model with spontaneous symmetry breakdown and shows the particle content in a manifest 
manner. The third gauge fixing condition, which we will name a "general gauge", is a linear combination of the Einstein gauge and the Weyl gauge [8], and will be argued in the next section. The final gauge condition was the "Lorenz gauge" $\nabla_{\mu} S^{\mu}=0$ which was used in evaluating an effective action of a quadratic gravity in the Weyl geometry [22].

Let us start with a quadratic gravity with the curvature squared and the kinetic term for the Weyl gauge field whose Lagrangian density is given by

$$
\mathcal{L}=\sqrt{-g}\left(\frac{\xi_{1}}{2} \tilde{R}^{2}-\frac{1}{4} H_{\mu \nu} H^{\mu \nu}\right),
$$

where a coupling constant $\xi_{1}$ is taken to be positive in order to avoid a ghost. The field equations for the Weyl gauge field $S_{\mu}$ and the metric field $g_{\mu \nu}$ can be obtained by putting $\phi=0$ in the field equations derived in the previous section. The field equation for the Weyl gauge field reads

$$
\nabla^{\nu} H_{\mu \nu}=6 \xi_{1} f D_{\mu} \tilde{R}
$$

The identity $\nabla^{\mu} \nabla^{\nu} H_{\mu \nu}=0$ then yields a relation

$$
\square \tilde{R}=2 f \nabla_{\mu}\left(S^{\mu} \tilde{R}\right)
$$

Moreover, the field equation for the metric tensor field leads to

$$
\begin{aligned}
& \xi_{1} \tilde{R}\left(\tilde{R}_{(\mu \nu)}-\frac{1}{4} g_{\mu \nu} \tilde{R}\right) \\
= & \frac{1}{2}\left(H_{\mu \alpha} H_{\nu}{ }^{\alpha}-\frac{1}{4} g_{\mu \nu} H_{\alpha \beta} H^{\alpha \beta}\right)+4 \xi_{1} f \tilde{R}\left[\nabla_{\mu} S_{\nu}-\frac{1}{4} g_{\mu \nu} \nabla_{\alpha} S^{\alpha}\right. \\
+ & \left.2 f\left(S_{\mu} S_{\nu}-\frac{1}{4} g_{\mu \nu} S_{\alpha} S^{\alpha}\right)\right]+6 \xi_{1} f\left[\frac{1}{2} g_{\mu \nu} \nabla_{\alpha}\left(S^{\alpha} \tilde{R}\right)-\nabla_{(\mu}\left(S_{\nu)} \tilde{R}\right)\right] \\
+ & \xi_{1}\left(\nabla_{\mu} \nabla_{\nu}-g_{\mu \nu} \square\right) \tilde{R} .
\end{aligned}
$$

Taking the trace of this equation gives us the same equation as in Eq. (41).

Next, we wish to consider a gauge invariant line element in the sense that it is invariant under both diffeomorphisms and the Weyl gauge transformation. It is of interest to see that there exists almost a unique candidate and one can construct such a line element without introducing a scalar field:

$$
d \tilde{s}^{2}=\tilde{R} g_{\mu \nu} d x^{\mu} d x^{\nu} \equiv \tilde{R} d s^{2} .
$$


Since the scalar curavature $\tilde{R}$ and the metric tensor $g_{\mu \nu}$ have weight -2 and 2 , respectively, a composite operator $\tilde{R} g_{\mu \nu}$ has weight 0 so it is invariant under the Weyl gauge transformation. Provided that the choice of a gauge condition makes the gauge invariant line element $d \tilde{s}$ coincide with the conventional line element $d s$ in the Riemann geometry (up to an overall constant), we are invited to choose the Weyl gauge condition

$$
\tilde{R}=k,
$$

where $k$ is a certain constant. Actually, with this gauge choice, the gauge invariant line element $d \tilde{s}$ reduces to that in the Riemann geometry as seen in Eq. (43). ${ }^{6}$

Before delving into a quantum theory, we would like to examine whether or not the Weyl gauge condition (44) allows a flat Minkowski metric $g_{\mu \nu}=\eta_{\mu \nu}$ as a classical solution. In particular, there might be a possibility that we could not find a Lorentz covariant solution for the Weyl gauge field. To show that there is indeed a Lorentz covariant solution, let us substitute the flat Minkowski metric into Eq. (44) whose result is rewritten as

$$
-6 f \partial_{\mu} S^{\mu}-6 f^{2} S_{\mu} S^{\mu}=k .
$$

As a Lorentz covariant solution, let us consider a pure gauge, $S_{\mu}=\partial_{\mu} \omega$ where $\omega$ is a scalar field. Inserting this pure gauge solution to Eq. (45) produces an equation

$$
-6 f \square \omega-6 f^{2}\left(\partial_{\mu} \omega\right)^{2}=k,
$$

with being $\square \equiv \eta^{\mu \nu} \partial_{\mu} \partial_{\nu}$. Assuming $\omega=c p_{\mu} x^{\mu}$ where $c$ is a constant and $p_{\mu}$ is a four dimensional momentum, Eq. (46) leads to a relation

$$
p_{\mu}^{2}=-\frac{k}{6 f^{2} c^{2}} \equiv-m^{2},
$$

where we have put $k=6(\mathrm{fcm})^{2}$, which holds only for a positive $k$. Actually, as will be seen later in Eq. (53), $k$ must be a positive constant. Thus, as long

\footnotetext{
${ }^{6}$ If we want a complete agreement between $d \tilde{s}$ and $d s$, we can change the definition of $d \tilde{s}^{2}$ like $d \tilde{s}^{2}=\frac{1}{k} \tilde{R} g_{\mu \nu} d x^{\mu} d x^{\nu}$.
} 
as $p_{\mu}$ satisfies the "mass-shell condition" (47), there is a Lorentz covariant solution for the Weyl gauge field, by which the flat Minkowski metric is a classical solution to the Weyl gauge condition (44).

Next, in order to move to a quantum theory, we rely on the BRST formalism. The BRST formalism can be made by two steps: The first step is to gauge-fix the Weyl gauge symmetry and the second step is to do diffeomorphisms. Since each BRST charge commutes, we will treat with only the BRST formalism for the Weyl gauge symmetry. The construction of the BRST formalism for diffeomorphisms can be done in a similar way to Ref. [28].

The BRST transformation for the Weyl gauge symmetry takes the form

$$
\begin{aligned}
\delta_{B} \sqrt{-g} & =4 c \sqrt{-g}, \quad \delta_{B} \tilde{R}=-2 c \tilde{R}, \\
\delta_{B} \bar{c} & =i B, \quad \delta_{B} B=\delta_{B} c=0
\end{aligned}
$$

where $c, \bar{c}$ and $B$ are a ghost, an anti-ghost and the Nakanishi-Lautrup field, respectively. Then, the Lagrangian density for the gauge fixing term and the FP ghost term is given by [29]

$$
\begin{aligned}
\mathcal{L}_{G F+F P} & =-i \delta_{B}\left[\sqrt{-g} \bar{c}\left(\tilde{R}-k+\frac{1}{2} \alpha B\right)\right] \\
& \left.=\sqrt{-g}\left[B_{*}(\tilde{R}-k)+\frac{1}{2} \alpha B_{*}^{2}-2 i k \bar{c} c\right)\right],
\end{aligned}
$$

where $\alpha$ is a gauge parameter and we have defined $B_{*} \equiv B+2 i \bar{c} c$. After performing the integration over $B_{*}, c, \bar{c}$, we have

$$
\mathcal{L}_{G F+F P}=-\frac{1}{2 \alpha} \sqrt{-g}(\tilde{R}-k)^{2}-i \hbar \delta^{4}(0) \log \sqrt{-g(x)} .
$$

The last term comes from the FP determinant and will be ignored in what follows since its existence does not change our results.

We wish to get the Einstein-Hilbert term from $\mathcal{L}$ in (39) plus $\mathcal{L}_{G F+F P}$ in (50). To do that, we first eliminate the $\tilde{R}^{2}$ term by choosing the gauge parameter $\alpha$ to be

$$
\alpha=\frac{1}{\xi_{1}} .
$$


With this gauge parameter we obtain a quantum Lagrangian density:

$$
\mathcal{L}_{q}=\sqrt{-g}\left(\xi_{1} k \tilde{R}-\frac{1}{4} H_{\mu \nu} H^{\mu \nu}-\frac{1}{2} \xi_{1} k^{2}\right) .
$$

Next, selecting the constant $k$ to be

$$
k=\frac{M_{P l}^{2}}{2 \xi_{1}},
$$

where $M_{P l}$ is the reduced Planck mass defined as $M_{P l}=\frac{1}{\sqrt{8 \pi G}}=2.44 \times$ $10^{18} \mathrm{GeV}$ and using (17), we arrive at the final expression up to a total derivative term:

$$
\mathcal{L}_{q}=\sqrt{-g}\left[\frac{M_{P l}^{2}}{2}(R-2 \Lambda)-\frac{1}{4} H_{\mu \nu} H^{\mu \nu}-\frac{1}{2} m_{S}^{2} S_{\mu} S^{\mu}\right],
$$

where the cosmological constant $\Lambda$ and the mass of the Weyl gauge field, $m_{S}$, are positive definite and given by

$$
\Lambda=\frac{1}{8 \xi_{1}} M_{P l}^{2}, \quad m_{S}=\sqrt{6} f M_{P l} .
$$

Here it is of interest to notice that the present formalism provides only a positive cosmological constant, which is very small at the weak coupling limit of the gravitational interaction, $\xi_{1} \gg 1$. Moreover, the mass of the Weyl gauge field depends on the coupling constant $f$ for the Abelian group.

In this way, starting with the classical Lagrangian density (39), we have performed the BRST quantization for the Weyl gauge symmetry by taking the Weyl gauge (44), and then succeeded in obtaining a quantum Lagrangian density (54), which is sum of the Einstein-Hilbert term plus the massive Weyl gauge field, by choosing the gauge parameter Eq. (51) and fix the constant $k=\frac{M_{P l}^{2}}{2 \xi_{1}}$. As a result of this process, we have spontaneous symmetry breakdown (SSB) of the Weyl gauge symmetry. However, it is unclear in the present gauge choice whether or not the number of dynamical degrees of freedom remains the same before and after the SSB, and what dynamical degree of freedom is eaten by the Weyl gauge field.

To answer these questions, it is convenient to move from the Jordan frame to the Einstein frame [30, 22]. First, let us rewrite the Lagrangian density 
(39) by introducing a scalar field $\varphi$ of weight -2 as follows:

$$
\mathcal{L}=\sqrt{-g}\left(\varphi \tilde{R}-\frac{1}{2 \xi_{1}} \varphi^{2}-\frac{1}{4} H_{\mu \nu} H^{\mu \nu}\right),
$$

where the field equation for $\varphi$ reads

$$
\varphi=\xi_{1} \tilde{R}
$$

Substituting Eq. (57) into Eq. (56) reproduces the classical Lagrangian density (39) as required.

In order to move to the Einstein frame, let us first invert (2):

$$
g_{\mu \nu}=\Omega^{-2}(x) \hat{g}_{\mu \nu},
$$

where we have defined $\Omega(x)=e^{\Lambda(x)}$. Various geometrical quantities can be rewritten in terms of $\hat{g}_{\mu \nu}$ in a new conformal frame.

$$
\begin{aligned}
g^{\mu \nu} & =\Omega^{2}(x) \hat{g}^{\mu \nu}, \quad \sqrt{-g}=\Omega^{-4}(x) \sqrt{-\hat{g}}, \\
\Gamma_{\mu \nu}^{\lambda} & =\hat{\Gamma}_{\mu \nu}^{\lambda}-\left(F_{\mu} \delta_{\nu}^{\lambda}+F_{\nu} \delta_{\mu}^{\lambda}-\hat{g}^{\lambda \rho} F_{\rho} \hat{g}_{\mu \nu}\right), \\
R & =\Omega^{2}(x)\left(\hat{R}+6 \square F-6 \hat{g}^{\mu \nu} F_{\mu} F_{\nu}\right),
\end{aligned}
$$

where we have defined

$$
\begin{aligned}
F & =\log \Omega, \quad F_{\mu}=\partial_{\mu} F=\frac{\partial_{\mu} \Omega}{\Omega}, \\
\square F & =\frac{1}{\sqrt{-\hat{g}}} \partial_{\mu}\left(\sqrt{-\hat{g}} \hat{g}^{\mu \nu} \partial_{\nu} F\right) .
\end{aligned}
$$

Using these expressions, one can rewrite the Lagrangian density (56) into a form

$$
\begin{aligned}
\mathcal{L} & =\sqrt{-\hat{g}}\left[\varphi \Omega ^ { - 2 } \left(\hat{R}-6 f \hat{\nabla}{ }_{\mu} S^{\mu}-6 f^{2} \hat{g}^{\mu \nu} S_{\mu} S_{\nu}+6 \square F-6 \hat{g}^{\mu \nu} F_{\mu} F_{\nu}\right.\right. \\
& \left.\left.+12 f \hat{g}^{\mu \nu} F_{\mu} S_{\nu}\right)-\frac{1}{2 \xi_{1}} \Omega^{-4} \varphi^{2}-\frac{1}{4} \hat{g}^{\mu \nu} \hat{g}^{\alpha \beta} H_{\mu \alpha} H_{\nu \beta}\right] .
\end{aligned}
$$

To reach the Einstein frame, we choose the scale factor $\Omega(x)$ such that

$$
\varphi \Omega^{-2}=\frac{1}{2} M_{P l}^{2}
$$


Then, up to total derivative terms, the Lagrangian density (61) is cast to an expression

$$
\begin{aligned}
\mathcal{L} & =\sqrt{-\hat{g}}\left(\frac{M_{P l}^{2}}{2} \hat{R}-3 f^{2} M_{P l}^{2} \hat{g}^{\mu \nu} \hat{S}_{\mu} \hat{S}_{\nu}-\frac{1}{8 \xi_{1}} M_{P l}^{2}\right. \\
& \left.-\frac{1}{4} \hat{g}^{\mu \nu} \hat{g}^{\alpha \beta} H_{\mu \alpha} H_{\nu \beta}\right),
\end{aligned}
$$

where we have defined

$$
\hat{S}_{\mu}=S_{\mu}-\frac{1}{2 f} \partial_{\mu} \log \varphi
$$

Note that this Lagrangian density (63) coincides with the previous one (54) with (55) (up to the FP ghost term).

The point of the above derivation is twofold. First, the Weyl gauge field $S_{\mu}$ "eats" a scalar field $\varphi$ as seen in Eq. (64), thereby the Weyl gauge field becoming massive, and the number of dynamical degrees of freedom remains the same before and after the SSB as expected. In other words, a scalar mode hidden in the curvature squared is absorbed into the longitudinal mode of the massless Weyl gauge field, by which the Weyl gauge field becomes massive. Second, the condition (62) corresponds to the "Einstein gauge" or "unitary gauge" for which the scalar field takes a constant value. Indeed, since the scalar field $\varphi$ has weight -2 , Eq. (62) means that in a new conformal frame, i.e., the Einstein frame, the scalar field takes a constant

$$
\varphi \rightarrow \hat{\varphi}=\Omega^{-2}(x) \varphi=\frac{1}{2} M_{P l}^{2}
$$

Incidentally, it turns out that the values of the cosmological constant and the mass of the Weyl gauge field in (55) are independent of the gauge choice so that they are physical quantities.

\section{A more general gauge and spontaneous sym- metry breakdown}

Encouraged by the observation in the previous section that spontaneous sym-

metry breakdown of the Weyl gauge symmetry occurs, we will search for the 
genuine spontaneous symmetry breakdown in the sense that a potential term determines the vacuum expectation value among arbitrary configurations.

Before doing so, it is worthwhile to summarize the SSB found in the previous section and point out its problem. In the latter part of the previous section, we have started with a quadratic gravity (39) in the Weyl geometry and then replaced it with a scalar-tensor gravity (56) which is quantum mechanically equivalent to the quadratic gravity (39). In the process of moving from the Jordan frame to the Einstein frame, in Eq. (62) we have chosen a dimensional constant, i.e., the Planck mass $M_{P l}$, to compensate for the dimension of mass squared of the scalar field $\varphi$. This change of the frames is found to be equivalent to taking the Einstein or unitary gauge. The introduction of the Planck mass into a Weyl invariant theory has triggered the SSB of the Weyl gauge symmetry. To put differently, the choice of the gauge condition has given rise to the SSB. This situation also holds in the arguments of the former part of the previous section where the choice of the Weyl gauge provided us with the SSB of the Weyl gauge symmetry.

Let us recall that in the conventional scenario of the SSB, there exists a potential inducing the SSB while we have no such potential in the theory under consideration. Nevertheless, the very presence of a solution with dimensional constants or the gauge condition for the Weyl gauge symmetry justifies the claim that the present scenario of the SSB is nothing but spontaneous symmetry breakdown. Actually, the SSB happens in such a way that one dynamical degree of freedom is "eaten" by a gauge field and as a result the gauge field becomes massive, and the number of dynamical degrees of freedom remains unchanged before and after the SSB.

A possible problem, however, arises from the lack of a suitable potential in the sense that we cannot single out a solution realizing the SSB on the stability argument [30]. In order to overcome this problem, we have derived an effective potential displaying the SSB from the radiative corrections of gravitational fields stemming from higher derivative terms $[20,22]^{7}$ by using the Coleman-Weinberg mechanism [32]. In this section, we would like to derive such a potential by using the gauge fixing condition. At first sight, it seems to be strange that one can derive a Higgs potential from the gauge fixing procedure since it is thought that the choice of a gauge fixing condition

\footnotetext{
${ }^{7}$ This study is based on our previous paper [31].
} 
is at one's disposal and physics does not usually depend on such a gauge condition. However, in case of scale invariant gravitational theories [33, 34, $35]$, the gauge fixing of a local scale symmetry or the Weyl gauge symmetry is usually associated with the introduction of dimensional constants (except for the Lorenz gauge condition), which triggers the SSB of the symmetry. In addition to it, our present study might provide a bold conjecture of the origin of the Higgs potential that the Higgs potential comes from a suitable gauge fixing for a local scale symmetry or the Weyl gauge symmetry.

We are now ready to start with a more general theory whose action is given in Eq. (32). Since $\tilde{R}$ and $\phi^{2}$ are invariant under diffeomorphisms and have weight -2 , one can construct a gauge invariant line element:

$$
\begin{aligned}
d \tilde{s}^{2} & =\left(a \tilde{R}+b \phi^{2}\right) g_{\mu \nu} d x^{\mu} d x^{\nu} \\
& \equiv\left(a \tilde{R}+b \phi^{2}\right) d s^{2}
\end{aligned}
$$

where $a$ and $b$ are constants. The requirement that this gauge invariant line element should be reduced to the line element in the Riemann geometry by taking a gauge condition gives us information on a more general gauge condition

$$
a \tilde{R}+b \phi^{2}=k
$$

where $k$ is a certain constant to be determined shortly.

Following the same line of the argument as before, let us use the BRST formalism to construct a quantum theory. In addition to the BRST transformation (48), we have a BRST transformation for $\phi$; since the scalar field $\phi$ has weight -1 , its BRST transformation is of form

$$
\delta_{B} \phi=-c \phi
$$

Then, the Lagrangian density for the gauge fixing term and the FP ghost term is given by

$$
\begin{aligned}
\mathcal{L}_{G F+F P} & =-i \delta_{B}\left[\sqrt{-g} \bar{c}\left(a \tilde{R}+b \phi^{2}-k+\frac{1}{2} \alpha B\right)\right] \\
& =-\frac{1}{2 \alpha} \sqrt{-g}\left(a \tilde{R}+b \phi^{2}-k\right)^{2}-i \hbar \delta^{4}(0) \log \sqrt{-g(x)}
\end{aligned}
$$


where we have performed the integration over $B_{*}, c, \bar{c}$ as before. The last term again comes from the FP determinant and will be ignored below.

Adding the classical Lagrangian density in (32), and the gauge-fixing and FP ghost Lagrangian density (69), we have

$$
\begin{aligned}
\mathcal{L}_{q} & =\sqrt{-g}\left\{\frac{1}{2}\left(\xi_{1}-\frac{a^{2}}{\alpha}\right) \tilde{R}^{2}+\left[\left(\frac{\xi_{2}}{2}-\frac{a b}{\alpha}\right) \phi^{2}+\frac{a k}{\alpha}\right] \tilde{R}\right. \\
& \left.-\frac{1}{4} H_{\mu \nu} H^{\mu \nu}-\frac{1}{2} g^{\mu \nu} D_{\mu} \phi D_{\nu} \phi-\frac{1}{2 \alpha}\left(b \phi^{2}-k\right)^{2}-\frac{\lambda}{4} \phi^{4}\right\} .
\end{aligned}
$$

To remove the term involving $\tilde{R}^{2}$, we shall take the gauge parameter $\alpha$ to be

$$
\alpha=\frac{a^{2}}{\xi_{1}} .
$$

With this choice of the gauge parameter, the Lagrangian density (70) becomes

$$
\begin{aligned}
\mathcal{L}_{q} & =\sqrt{-g}\left\{\left[\left(\frac{\xi_{2}}{2}-\frac{\xi_{1} b}{a}\right) \phi^{2}+\frac{\xi_{1} k}{a}\right] \tilde{R}-\frac{1}{4} H_{\mu \nu} H^{\mu \nu}\right. \\
& \left.-\frac{1}{2} g^{\mu \nu} D_{\mu} \phi D_{\nu} \phi-\frac{\xi_{1}}{2 a^{2}}\left(b \phi^{2}-k\right)^{2}-\frac{\lambda}{4} \phi^{4}\right\} \\
& =\sqrt{-g}\left\{\left[\left(\frac{\xi_{2}}{2}-\frac{\xi_{1} b}{a}\right) \phi^{2}+\frac{\xi_{1} k}{a}\right] \tilde{R}-\frac{1}{4} H_{\mu \nu} H^{\mu \nu}\right. \\
& -\frac{1}{2} g^{\mu \nu} D_{\mu} \phi D_{\nu} \phi-\frac{\xi_{1}}{2 a^{2}}\left(b^{2}+\frac{a^{2} \lambda}{2 \xi_{1}}\right)\left(\phi^{2}-\frac{b k}{b^{2}+\frac{a^{2} \lambda}{2 \xi_{1}}}\right)^{2} \\
& \left.-\frac{k^{2} \lambda}{4\left(b^{2}+\frac{a^{2} \lambda}{2 \xi_{1}}\right)}\right\} .
\end{aligned}
$$

It is worthwhile to notice that the Higgs potential has emerged in the Lagrangian density from the gauge fixing condition (67) for the Weyl gauge transformation due to $\xi_{1}>0, \lambda>0$. The present theory, therefore, solves the "possible' problem" mentioned above and the presence of the Higgs potential makes it possible to single out a solution realizing the SSB on the stability argument. 
Taking the vacuum expectation value

$$
\left\langle\phi^{2}\right\rangle=\frac{b k}{b^{2}+\frac{a^{2} \lambda}{2 \xi_{1}}},
$$

we have a quantum Lagrangian density in the lowest level of approximation

$$
\begin{aligned}
\mathcal{L}_{q} & =\sqrt{-g}\left[\frac{k\left(\xi_{2} b+a \lambda\right)}{2\left(b^{2}+\frac{a^{2} \lambda}{2 \xi_{1}}\right)} \tilde{R}-\frac{1}{4} H_{\mu \nu} H^{\mu \nu}\right. \\
& \left.-\frac{1}{2} \frac{f^{2} b k}{b^{2}+\frac{a^{2} \lambda}{2 \xi_{1}}} S_{\mu} S^{\mu}-\frac{k^{2} \lambda}{4\left(b^{2}+\frac{a^{2} \lambda}{2 \xi_{1}}\right)}\right] .
\end{aligned}
$$

To make this Lagrangian density coincide with the Einstein-Hilbert Lagrangian, we choose the constant $k$ to be

$$
k=\frac{b^{2}+\frac{a^{2} \lambda}{2 \xi_{1}}}{\xi_{2} b+a \lambda} M_{P l}^{2} .
$$

As a result, up to a total derivative term, (74) takes the form

$$
\begin{aligned}
\mathcal{L}_{q} & =\sqrt{-g}\left[\frac{M_{P l}^{2}}{2} R-\frac{1}{4} H_{\mu \nu} H^{\mu \nu}-\frac{1}{2} \frac{\left(6 \xi_{2}+1\right) b+6 a \lambda}{\xi_{2} b+a \lambda}\left(f M_{P l}\right)^{2} S_{\mu} S^{\mu}\right. \\
& \left.-\frac{\lambda\left(b^{2}+\frac{a^{2} \lambda}{2 \xi_{1}}\right)}{4\left(\xi_{2} b+a \lambda\right)^{2}} M_{P l}^{4}\right] .
\end{aligned}
$$

From this Lagrangian density, one can easily read out the values of the cosmological constant and the mass of the Weyl gauge field as

$$
\begin{aligned}
\Lambda & =\frac{\lambda\left(b^{2}+\frac{a^{2} \lambda}{2 \xi_{1}}\right)}{4\left(\xi_{2} b+a \lambda\right)^{2}} M_{P l}^{2}, \\
m_{S} & =\sqrt{\frac{\left(6 \xi_{2}+1\right) b+6 a \lambda}{\xi_{2} b+a \lambda}} f M_{P l} .
\end{aligned}
$$

Note that the the cosmological constant is again positive definite and very tiny in the weak coupling regime $\xi_{2} \gg 1, \lambda \ll 1 .^{8}$ Of course, the number of dynamical degrees of freedom is unchanged before and after the SSB, and a scalar field in the scalar curvature squared is eaten by the massless Weyl gauge field as in the previous model.

\footnotetext{
${ }^{8}$ Even if the cosmological constant is tiny in the weak coupling limit, this is no longer a solution to the cosmological constant problem as proved in Refs. [36, 37, 38, 39].
} 


\section{Conclusions}

In this article, we have investigated a possibility that gravitational theories in the Weyl geometry, which are invariant under the Weyl gauge transformation as well as diffeomorphisms, generate the Einstein-Hilbert action of Einstein's general relativity. We have seen that a quadratic gravity without a scalar field induces the Einstein-Hilbert action and at the same time the Weyl gauge field becomes massive in the "Weyl gauge" where Weyl's scalar curvature takes a constant value. Furthermore, we have showed that the same phenomenon also occurs in the "Einstein gauge" or "unitary gauge" for which the scalar field takes a constant value. The latter gauge choice clarifies that a scalar field included in the scalar curvature squared is absorbed into a longitudinal mode of the massless Weyl gauge field, thereby the gauge field becoming massive. We also pointed out that this phenomenon is nothing but spontaneous symmetry breakdown (SSB) $[30,22]$ in the sense that the gauge field becomes massive and the number of physical degrees of freedom remains unchanged before and after the SSB, but the absence of the Higgs potential provides us a problem that one cannot single out a solution realizing the SSB on the stability argument.

In order to solve the problem of the absence of the Higgs potential, we have looked for a more general model of a quadratic gravity with a scalar field in the Weyl geometry. This general model in fact shows an interesting phenomenon that a more general gauge fixing condition yields a Higgs potential whose minimum determines the vacuum expectation value, thereby triggering spontaneous symmetry breakdown. In this model, the EinsteinHilbert term is also induced and the Weyl gauge field becomes massive via the SSB.

The key observation behind the present work is to overcome the second clock problem associated with the Weyl geometry. The second clock problem says that two synchronized clocks put at a certain space-time point run at different speeds after they travel along different paths and then reunite at some place. To put differently, two identical elementary particles with the same mass show different masses after they get the same experience as above since mass is nothing but length scale in this context. However, in this interpretation, there is an implicit assumption such that the length $l$ has a 
variation

$$
d l=f l S_{\mu} d x^{\mu}
$$

and its integration $l$ given by

$$
l=l_{0} e^{f \int S_{\mu} d x^{\mu}},
$$

determines a physcal length. As long as we admit this implicit assumption about the physical length, we are forced to encounter the second clock problem. One famous resolution, which eventually led to the concept of the $U(1)$ gauge invariance, is to replace the scale factor $f$ with a imaginary phase if . Then, the second clock problem amounts to the question of whether, say, two electrons starting with the same phase and taken along different paths would acquire different phases or not. The experimental answer is definitely yes and this phenomenon is nothing but the Aharonov-Bohm effect.

An alternative resolution, which we adopt in this article, is that we do not regard $d s^{2}=g_{\mu \nu} d x^{\mu} d x^{\nu}$ or $l$ as a physical quantity measuring the distance since it is not gauge invariant. The gauge invariant line element $d \tilde{s}$ defined in Eq. (43) or Eq. (66) should be regarded as the definition of the distance in the Weyl geometry. It is then obvious that we are free from the second clock problem. If we require that this gauge invariant line element $d \tilde{s}$ is reduced to $d s$ in the Riemann geometry after fixing the Weyl gauge invariance, we are led to taking the gauge condition, the Weyl gauge or the more general gauge.

To close this article, let us comment on the Higgs potential. Let us recall that there is a longstanding and important question of what the origin of the Higgs potential is. Our model might partially answer this question: The Higgs potential comes from symmetry breaking of a local scale symmetry or Weyl gauge symmetry. In this context, it is worth recalling that the standard model is invariant under a scale symmetry if the (negative) mass term for the Higgs field is removed from its action [40]. In this sense, it is naturally expected that the appearance of the Higgs potential is closely related to the scale symmetry. The Weyl geometry provides us with a playground for promoting a global scale symmetry to a local scale symmetry and simultaneously produces a Weyl gauge field as a candidate for dark matter. 


\section{References}

[1] H. Weyl, "Gravitation und Elekrizität", Sitzungsberichte der Königlich Preußischen Akademie der Wissenschaften zu Berlin, 1918, pp. 465480; English translation, "Gravitation and Electricity", pp. 24-37 in O'Raifeartaigh's book.

[2] R. Penrose, "The Road to Reality", Vintage Books, New York, 2007.

[3] F. London, "Quantum-Mechanical Interpretation of Weyl's Theory", Zeit. Phys. 42 (1927) 375.

[4] L. O'Raifeartaigh, "The Dawning of Gauge Theory", Princeton University Press, 1997.

[5] M. Omote, "Scale Transformations of the Second Kind and the Weyl Space-Time", Lett. Nuovo. Cim. 2 (1971) 58.

[6] P. A. M. Dirac, "Long Range Forces and Broken Symmetries", Proc. Roy. Soc. Lond. A 333 (1973) 403.

[7] R. Utiyama, "On Weyl's Gauge Field", Prog. Theor. Phys. 50 (1973) 2080 .

[8] P. G. O. Freund, "Local Scale Invariance and Gravitation", Ann. of Phys. 84 (1974) 440.

[9] R. Utiyama, "On Weyl's Gauge Field II", Prog. Theor. Phys. 53 (1975) 565.

[10] K. Hayashi, M. Kasuya and T. Shirafuji, "Elementary Particles and Weyl's Gauge Field", Prog. Theor. Phys. 57 (1977) 431; Erratum: Prog. Theor. Phys. 59 (1978) 681.

[11] K. Hayashi and T. Kugo, "Remarks on Weyl's Gauge Field", Prog. Theor. Phys. 61 (1979) 334.

[12] L. Smolin, "Towards a Theory of Spacetime Structure at Very Short Distances", Nucl. Phys. B 160 (1979) 253. 
[13] H. Cheng, "Possible Existence of Weyl's Vector Meson", Phys. Rev. Lett. 61 (1988) 2182.

[14] W. Drechsler and H. Tann, "Broken Weyl Invariance and the Origin of Mass", Found. Phys. 29 (1999) 1023.

[15] H. Nishino and S. Rajpoot, "Implication of Compensator Field and Local Scale Invariance in the Standard Model", Phys. Rev. D 79 (2009) 125025 .

[16] C. Pagani and R. Percacci, "Quantization and Fixed Points of NonIntegrable Weyl Theory", Class. Quant. Grav. 31 (2014) 115005.

[17] H. C. Ohanian, "Weyl Gauge-vector and Complex Dilaton Scalar for Conformal Symmetry and Its Breaking", Gen. Rel. Grav. 48 (2016) 25.

[18] M. de Cesare, J. W. Moffat and M. Sakellariadou, "Local Conformal Symmetry in Non-Riemannian Geometry and the Origin of Physical Scales", Eur. Phys. J. C 77 (2017) 605.

[19] D. M. Ghilencea, "Spontaneous Breaking of Weyl Quadratic Gravity to Einstein Action and Higgs Potential", JHEP 1903 (2019) 049.

[20] I. Oda, "Planck and Electroweak Scales Emerging from Weyl Conformal Gravity", arXiv:1903.09309 [hep-th], To appear PoS, The Corfu Summer Institute 2018, Workshop on the Standard Model and Beyond.

[21] D. M. Ghilencea, "Stueckelberg Breaking of Weyl Conformal Geometry and Applications to Gravity", Phys. Rev. D 101 (2020) 045010.

[22] I. Oda, "Planck Scale from Broken Local Conformal Invariance in Weyl Geometry", Adv. Studies in Theor. Phys. 14 (2020) 9.

[23] T. A. T. Sanomiya, I. P. Lobo, J. B. Formiga, F. Dahia and C. Romero, "An Invariant Approach to Weyl's Unified Field Theory", arXiv:2002.00285 [gr-qc].

[24] E. Scholz, "The Unexpected Resurgence of Weyl Geometry in late 20thCentury Physics", Einstein Stud. 14 (2018) 261. 
[25] R. Adler, M. Bazin and M. Schiffer, "Introduction to General Relativity", McGraw-Hill Inc., 1975.

[26] R. M. Wald, "General Relativity", University of Chicago Press, Chicago, 1984.

[27] K. S. Stelle, "Renormalization of Higher Derivative Quantum Gravity", Phys. Rev. D 16 (1977) 953.

[28] N. Nakanishi and I. Ojima, "Covariant Operator Formalism of Gauge Theories and Quantum Gravity, World Scientific Publishing, 1990, and references therein.

[29] T. Kugo and S. Uehara, "General Procedure of Gauge Fixing Based on BRS Invariance Principle", Nucl. Phys. B 197 (1982) 378.

[30] Y. Fujii and K. Maeda, "The Scalar-Tensor Theory of Gravitation", Cambridge University Press, 2003.

[31] I. Oda, "Planck and Electroweak Scales Emerging from Conformal Gravity", Eur. Phys. J. C 78 (2018) 798.

[32] S. R. Coleman and E. J. Weinberg, "Radiative Corrections as the Origin of Spontaneous Symmetry Breaking", Phys. Rev. D 7 (1973) 1888.

[33] I. Oda, "Classically Scale-invariant B-L Model and Dilaton Gravity", Phys. Rev. D 87 (2013) 065025.

[34] I. Oda, "Classically Scale-invariant B-L Model and Conformal Gravity", Phys. Lett. B 724 (2013) 160.

[35] I. Oda, "Higgs Mechanism in Scale-Invariant Gravity", Adv. Studies in Theor. Phys. 8 (2014) 215.

[36] S. Weinberg, "The Cosmological Constant Problem", Rev. Mod. Phys. 61 (1989) 1.

[37] I. Oda, "Weinbergs No Go Theorem in Quantum Gravity", Phys. Rev. D 96 (2017) 124012. 
[38] M. Nagahama and I. Oda, "More on Weinbergs No-go Theorem in Quantum Gravity", Phys. Rev. D 97 (2018) 104043.

[39] I. Oda, "Scale Symmetry and Weinberg's No-go Theorem in the Cosmological Constant Problem", Adv. Studies in Theor. Phys. 13 (2019) 195.

[40] W. A. Bardeen, "On Naturalness in the Standard Model", FERMILABCONF-95-391 (1995). 